\title{
Technological evolution of diagnostic testing in
}

\section{oncology}

Diagnostic testing has been improving the quality of cancer care. The dynamics of this field can be grasped through the application of innovation lifecycle models. Single testing, parallel testing and whole-genome sequencing are major technological evolutions. Given the increasing availability of biomarkers, the performance of single testing will be limited in the future, favoring the further implementation of parallel testing technologies. Whole-genome sequencing will lead to a further performance increase by introducing the era of genomic medicine. A broad adoption of presently available diagnostic technologies sets up the infrastructure for future technologies. The speed at which these technologies are introduced depends heavily on the regulatory and reimbursement environment, while their final diffusion is subject to pragmatic criteria such as user friendliness, perceived risk and perceived value added.

\section{KEYWORDS: diagnostic testing medical oncology molecular diagnostic techniques multiplex assays personalized medicine technology S-curve whole-genome sequencing}

The field of oncology has been undergoing a major paradigm shift, as it has been moving away from the 'one-size-fits-all' approach to a 'test-and-treat' strategy brought about by personalized medicine. The personalization of cancer treatments is associated with various benefits compared with traditional treatments. These include increased efficacy, fewer adverse events, improved quality of life and an efficient use of healthcare expenditure [1-3,101]. Within the overall framework of personalized medicine, diagnostic tests play a key role and can generally be described as a technology used to identify the right treatment for the right patient at the right time. Just like any other technology, diagnostics follow the basic rules of innovation lifecycles. One of the most famous theories used to explain the evolution of technologies is Moore's law published by Moore in 1965. Moore noted that the number of transistors on a computer chip doubled every 18 months during a 5 year period and concluded that this linear performance improvement will proceed in the future, as new knowledge will continuously be generated and processes will further be improved [4]. The insights delivered by Moore can be supplemented by other theories such as the S-curve concept, which describes how the performance of a technology develops over time [5], or Roger's diffusion curve, which analyzes the rate at which innovations are adopted in the market [6]. Applying these concepts to the field of diagnostic testing in oncology helps us to understand and foresee the evolution of this crucial technology in current and future cancer treatments.

\section{Technology S-curves}

The S-curve concept measures the performance of a certain technology over time. Performance can be defined as the accomplishment of a task measured against standards of accuracy, completeness, cost and speed [102]. A technology S-curve goes through four different phases [5]:

- Phase 1: introduction, the technology is introduced to the market and its performance level is low;

- Phase 2: development, improvements of the technology lead to a sharp performance increase;

- Phase 3: maturation, the technology reaches its performance limit;

- Phase 4: aging, fundamental physical constraints lead to a deteriorating performance.

Applying the S-curve concept to the topic of diagnostic testing in oncology suggests that the emergence of new technologies will lead to a significant overall performance increase of diagnostic testing throughout the next few years (Figure 1).

\section{S-curve 1: single testing}

The first S-curve represents companion diagnostic technologies. Companion diagnostics determine whether a patient is likely to respond to a

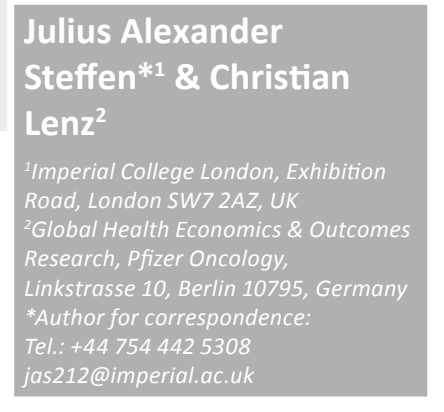




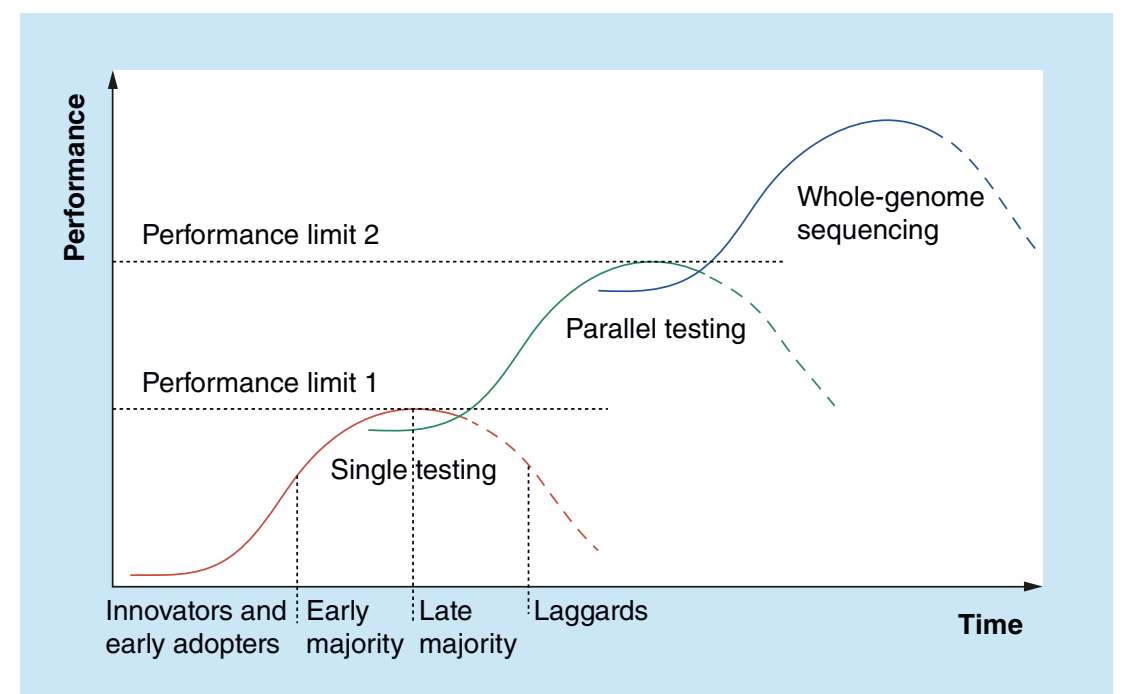

Figure 1. Technology S-curves of diagnostic testing. Demonstrates the application of the S-curve concept and Roger's diffusion curve [6] to diagnostic testing in oncology. A certain share of practitioners do not succeed in adopting the current testing technology before it reaches the aging phase. This is an impediment to the introduction of new technologies. certain treatment $[7]$. This approach implies that each treatment is 'accompanied' by a respective biomarker and diagnostic test. The measurement of companion biomarkers helps physicians make a better treatment decision and, thus, improves the health of a patient [103]. Among the variety of existing companion biomarkers, including protein and metabolite markers, the technologies described in this article make use of genetic biomarkers. As companion diagnostics must be conducted ahead of the treatment prescription, this approach is often characterized as a 'test-and-treat strategy' and is opposed to the 'trial-and-error' or 'one-size-fits-all' approach of conventional therapies $[3,104]$.

Companion diagnostics are linked to the concept of single testing, in which a proposed hypothesis is either confirmed or excluded through testing for mutations in a specific gene that can explain the disease. Patients are tested for different biomarkers in a sequential stepby-step way: if biomarker $A$ tests negative then biomarker B is tested; if negative, biomarker $\mathrm{C}$ is tested, and so on [8]. Diagnostic testing in nonsmall-cell lung cancer, for instance, often occurs in a sequential way; pathologists frequently test for $K R A S$ mutations first as these occur most frequently. If patients test negative for $K R A S$, they are then tested for EGF receptor (EGFR) and, if negative, they are tested for anaplastic lymphoma kinase (ALK). If negative, then these triple-negative tumors are considered for analysis of rarer mutations (e.g., in $B R A F, M E K 1, A K T 1$ and $P I K 3 C A)[9]$.
Currently available companion diagnostic technologies fall into different places along the $S$-curve. ALK testing in non-small-cell lung cancer, for instance, gained US FDA approval in 2012 and is, therefore, still in the introduction phase. The $S$-curve concept suggests that ongoing improvements of ALK-testing technologies will cause a sharp performance increase of ALK testing in the future. Her2/neu testing in breast cancer, on the other hand, has been on the market for a considerable amount of time and was first approved with Herceptin ${ }^{\circledR}$ (Genentech Inc., CA, USA) in 1998 [105]. This test has undergone various improvements and has most likely achieved its performance limit, which is why it can be allocated to the maturity phase.

\section{- Growing biomarker availability}

The S-curve concept suggests that, at later stages, technologies will face fundamental physical constraints, which will introduce the final aging stage [5]. In the case of single testing, this constraint will be posed by a growing availability of biomarkers.

The validation of biomarkers has been increasingly emphasized in clinical research activities $[10,104,106]$. This has been highlighted in a study analyzing the application of biomarkers in clinical trials registered on ClinicalTrials.gov [104], whereas prior to the year 1990, biomarkers were applied in only $4 \%$ of clinical studies. This share increased to $20 \%$ for the years after 2005 and is expected to further rise in the future [104]. This complies with other expert views predicting a compound annual biomarker growth rate of 25-30\% [106]. As more biomarkers are becoming available, treatment success will be greater and more often assessable in advance, which is why experts suggest that $20 \%$ of future drugs will be accompanied by a respective diagnostic test [106]. The trend towards an increasing availability and application of biomarkers has been characterized as 'biomarkerization' [10].

The growing availability of biomarkers renders the approach of single diagnostic testing more and more challenging. As each biomarker is tested successively, the number of tests that can possibly be conducted in a single patient grows significantly. This leads to a higher consumption of tissue and time, resulting from multiple physician appointments and various tests that are ordered [8].

A practical example of problems arising from the increasing availability of biomarkers is given by the topic of tissue management. Medical oncologists often report issues with having 
enough tissue samples for molecular testing, which has been repeatedly reported to delay proper patient screening [11-13]. As oncologists frequently have to request additional tumor biopsies, the testing process is considerably delayed. The growing number of available biomarkers will make the existent issue of providing a sufficient amount of tissue become increasingly difficult in the future.

The trend towards biomarkerization consequently limits the performance increase of single testing and causes physical constraints that will lead to the aging of these technologies. An example is given by Sanger sequencing, which is entering the aging phase because of its limited scalability [14]. This technology has been heavily used over the past 30 years and is still an integral part of practice [15]. Technological progress, however, has triggered the development of parallel tests that respond to the increasingly complex testing environment in a clearly more efficient way. Yet, for a balanced view, it is equally important to recognize that some technologies may not follow the $S$-curve principle. Immunohistochemistry, for example, is very likely to prove 'immortal' and be part of future S-curves as well.

\section{S-curve 2: parallel testing}

Parallel testing can occur in three different ways, each leading to clear improvements compared with single-testing technologies:

- Testing for one concrete biomarker in various samples, with one biomarker per sample;

- Testing for various biomarkers in various samples, with one biomarker per sample;

- Testing for various biomarkers in a single sample.

Considering the fast increase in biomarker availability, parallel testing leads to a significant performance increase of diagnostic testing as reflected by the second S-curve.

The parallel evaluation of disease-associated genes increases the efficiency of diagnostic testing while decreasing its complexity. Under single testing, the discovery of novel biomarkers increases the number of tests that can possibly be conducted per patient. Under parallel testing, however, a new biomarker can simply be added as a further analyte, which increases the complexity of a single test run but barely affects overall costs. This leads to concrete savings in terms of material and time (Figure 2). Proponents of parallel testing, therefore, state that this technology "drastically reduces the time and cost of diagnosis while maintaining accuracy and reliability" $[8]$.

The largest improvement as opposed to single testing is provided by the third way of parallel testing. A concrete example of this is given by multiplex assays, examining the status of multiple variants within a single sample. By measuring multiple targets in a single test run [8,15], multiplex assays make the parallel assessment of up to 384 genetic markers possible [8]. Multiplex assays are most likely to lead to a profound efficiency increase if they are systematically organized, for example, by focusing on the most frequently occurring mutations rather than on all possible genetic alternations. A further advantage over single testing is that mutations that are associated with disease groups marked by overlapping symptoms can be tested in a single test run. Through this, the parallel assessment of biomarkers delivers a value for physicians of different specialties [8].

Besides the economic benefits of multiplex testing, it also has positive implications for the patient's disease status. The faster a diagnostic test result is received, the more favorable the effects on the patient's health. The time to make an adequate therapeutic decision is shortened, which can reduce the uncertainty and anxiety experienced by the patient and his or her social environment [8].

The feasibility of multiplex testing in a general hospital setting has been proven in a study conducted at the Massachusetts General

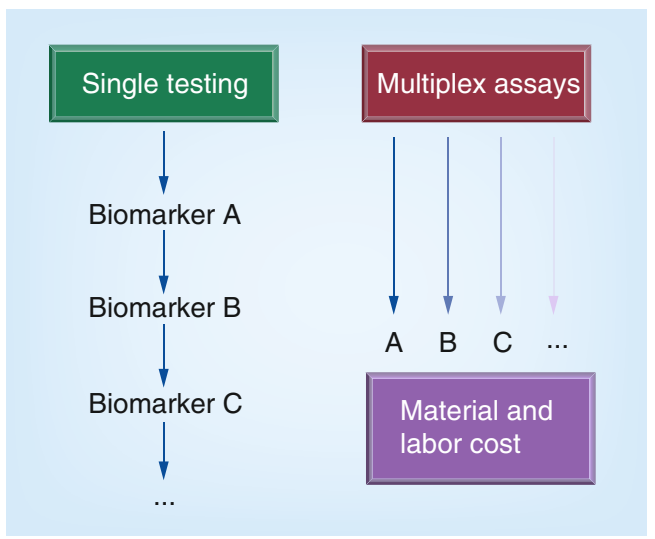

Figure 2. Benefits of parallel testing compared with single testing. In single tests, adding a further analyte increases the complexity of diagnostic testing vertically, leading to a higher consumption of material and time. Under multiplex testing, an additional analyte increases the complexity of a single test run horizontally, but barely affects the overall consumption of material and time. 
Hospital Cancer Center (MA, USA), which developed a multiplexed PCR-based assay that simultaneously identifies more than 50 nonsmall-cell lung carcinoma-specific mutations [16]. The data of the first 589 patients referred for genotyping suggests that multiplex testing can be incorporated into clinical routine and is, therefore, likely to become an integral part of practice as the availability of targeted therapies increases [16].

Concerning its position on the S-curve, multiplex testing is increasingly being implemented into clinical routine, suggesting that in many countries it has left the introduction phase and is experiencing a fast performance increase during the development phase. Yet, as a characteristic of this stage, various improvements still need to be made. A key challenge remains the provision of a proper infrastructure. This is an issue that has been addressed by leading institutions such as the Center for Integrated Oncology in Cologne, Germany [17,107]. To benefit from emerging technologies in the future, the creation of a facilitating environment must start today by implementing presently available diagnostic tests into clinical routine.

\section{S-curve 3: whole-genome sequencing}

The third S-curve of whole-genome sequencing reflects the basic assumptions of Moore's law, since the major driving force behind this approach is a sharp fall in the costs of sequencing the human genome [18].

There are 20,000-25,000 genes in the human genome, which consists of 3 billion chemical base pairs that make up human DNA and account for a data capacity of 3 GB [108]. The identification of a complete set of human DNA in 2003 triggered an 'explosion of investigation' and led to fast technological progress [101,19]. As a result, the cost of sequencing an entire human genome declined at the rate described by Moore's law. Accordingly, the decreasing costs of whole-genome sequencing led to a continuous doubling of efficiency [101]. Costs fell from US \$100-300 million in 2001 to US\$10 million in 2007 [109]. In spite of a decrease of at least $90 \%$ within 6 years, sequencing costs remained at a level that could exclusively be accessed by highly funded laboratories or government initiatives [101].

This complies with the suggestions made by the S-curve concept: once the human genome had been entirely sequenced, the invention had been made and was theoretically available as a new approach to diagnostic testing. However, its high cost made a broad implementation impossible, which is why the introduction phase could not yet be reached.

Around mid-2007, next-generation sequencing (NGS), producing a larger output within a shorter time frame [101,108], led to a major turn in events: "the ability to sequence entire genomes accelerated by a rate far exceeding that ever experienced by the semiconductor and computer industries" [101]. As the sequencing process becomes parallelized, NGS technologies can produce millions of sequences at once [20], and thus lead to economies of scale: the sequencing output per time increases while cost per unit of output decrease. An example of a successful NGS technologies is the Illumina ${ }^{\circledR}$ (CA, USA) genome analyzer platform using an array technique that makes parallel sequencing of millions of DNA fragments possible with a run time of 1.5-8 days [14].

As a result, Moore's law no longer applied and DNA sequencing is, in fact, considered the technology that has been accelerating most quickly throughout the last decade [101]. At the beginning of 2012, the 'less than US $\$ 1000$ genome', which may be considered the ultimate milestone on the path towards implementing whole-genome sequencing, has become reality. In 2012, Illumina and Life Technologies (CA, USA) released genome-sequencing machines capable of decoding the entire human DNA at less than US $\$ 1000$. Illumina's MiSeq ${ }^{\circledR}$ integrates amplification, sequencing and data analysis in a single instrument and makes it possbile to go from sample to data in $8 \mathrm{~h} \mathrm{[110].} \mathrm{Similarly,}$ Life Technologies' Ion Torrent ${ }^{\mathrm{TM}}$ increases the accessibility to genome sequencing owing to low cost and a sequencing run time of $2-4 \mathrm{~h}$ [111].

The realization of the less than US $\$ 1000$ genome made whole-genome sequencing become a real alternative to existing diagnostic testing approaches [108]. At such a cost, wholegenome sequencing will no longer be limited to well-funded projects, but can increasingly be embedded into clinical practice. This will allow physicians to treat each patient according to his or her individual genetic makeup, and will be a major step towards truly personalized medicine [108,21].

The implementation of whole-genome sequencing thus leads to a further performance increase within the field of diagnostic testing as visualized by $S$-curve 3 . The performance increase is driven by new opportunities becoming apparent to the diagnosis of cancer [101,112]. 
By analyzing the entire DNA, whole-genome sequencing identifies all genetic features in a single test (Figure 3), which will accelerate the growth in cancer gene discovery and will deliver novel targets for a large number of cancer types [21].

Two studies conducted by researchers at the Washington University in St Louis (MO, USA) have underscored the value of using wholegenome sequencing as a diagnostic tool [112]. By sequencing patients' entire genomes, they were able to discover novel genetic alternations that were having an impact on the patients' disease status and helped physicians adjust the course of treatment accordingly. With conventional tests and targeted sequencing approaches, these findings would not have been made. A concrete case described in the study involves a 39-year-old woman who was diagnosed with acute myeloid leukemia and who was recommended a stem cell treatment, since previous tests had indicated low survival. Sequencing her genome, however, led to the discovery of a novel genetic mutation, which suggested a change in the patient's treatment. Based on the sequencing results, the patient was treated with a targeted chemotherapy regimen, which eventually led to remission [112]. The benefits of whole-genome sequencing has been confirmed in a recent study by Iyer and colleagues [22] and is the reason why proponents suggest that whole-genome sequencing is a universal diagnostic approach delivering a complete picture of health and disease at an individual level [22-24].

Both studies reflect the performance increase realized in diagnostic testing through the adoption of whole-genome sequencing. In fact, the emergence of whole-genome sequencing has the potential to introduce an entirely new era of medicine extending the scope of traditional treatments significantly. The demand for genomic healthcare might eventually limit the performance increase of multiplex testing. As the abovementioned example at Washington University suggests, genome sequencing delivers results that go beyond the capacities of multiplex assays and are, therefore, likely to trigger a third S-curve.

\section{Diffusion of testing technologies}

The S-curve concept helps us to understand the emergence of innovative approaches to diagnostic testing in oncology. At what speed innovations are adopted by the market is explained by the Diffusion of Innovations Theory, published by Everett Rogers in 1962. It states that

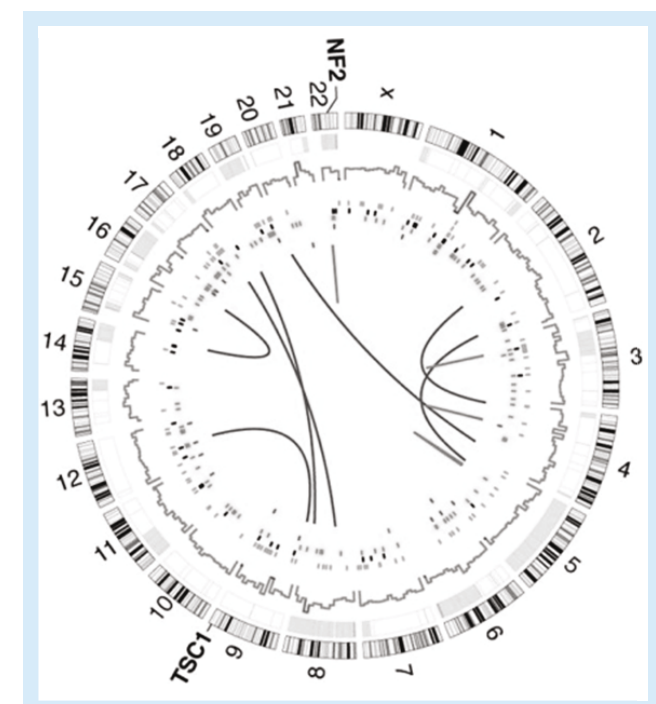

Figure 3. Identification of genetic alternations through whole-genome sequencing. Sequencing the human genome leads to the identification of all genetic abnormalities at once. The different shades indicate the existence of mutations such as deletion mutations or intra- and interchromosomal rearrangements.

Reproduced with permission from [21] (C) American Association for the Advancement of Science (2009).

various groups adopt innovations at a different points in time. Rogers distinguishes between the following groups [6]:

" Innovators and fast followers: adopt a technology early after its introduction, make it acceptable and spread its benefits via word-of-mouth;

- Early majority: represent a mainstream group that adopts a technology after observing innovators and fast followers;

- Late majority: low affinity to innovations and may adopt new technologies because previously used technologies are no longer available;

- Laggards: adopt a technology when innovators have most likely moved on.

Supplementing the S-curve concept with Roger's diffusion curve points out that a significant proportion of the market does not succeed in adopting an innovation before it is replaced by a novel technology (Figure 1). It is consequently questionable whether the infrastructure for subsequent technologies exists once they become available. To maximize the benefits and availability of upcoming innovations in diagnostic testing, it is, therefore, vital to implement current testing approaches in clinical practice and 
build a broad diagnostic testing infrastructure as early and widely as possible.

The diffusion of innovation concept states that there are general factors affecting the rate of adoption [6]. These include the fit with user needs, perceived risk, perceived value added and user friendliness. The practical validity of these factors can be shown by the example of EGFR mutation analysis through Sanger sequencing in lung carcinomas. Although, nowadays, this technology is widely spread and well established, achieving this status required the initial consideration of all four aspects listed above. Early versions of this technology were slow and surrounded by an overall debate on whether diagnostic testing was necessary at all [25]. The user need for EGFR testing was low, which resulted in a slow rate of diffusion. In addition, risk aversion slowed down the diffusion of EGFR-testing technologies: oncologists favored empirical therapies with erlotinib, as these were thought to involve a low level of risk for patients without EGFR mutations [25]. As well as this, the perceived value added of this technology decreased as a growing amount of data suggested that FISH and immunohistochemistry were at least as predictive [25]. Finally, Sanger sequencing did not allow for the testing of samples with only low amounts of tumor [25], which led to a low level of user friendliness.

An extensive adoption of testing technologies requires a maximization of user friendliness and perceived value added. Consequently, technical problems associated with a given diagnostic test need to be resolved. In the case of companion diagnostics, for instance, a limited availability of tissue calls for a more streamlined and standardized approach to specimen acquisition and processing [26]. Tissue handling, processing and sectioning must be standardized in order to minimize wastage, and optimize use of tissue for staining procedures and molecular tests [13]. Furthermore, rigorous quality assessment to ensure reliable results and appropriate patient selection must be introduced [13].

In addition to this, the diffusion of technologies represented by the second and third $S$-curves particularly has not sufficiently taken place because some providers lack awareness and genetics-related skills [27]. In order to make sure that the value of new sequencing technologies will be unlocked in clinical practice, healthcare professionals need to be educated and trained $[28,29]$. Not only practicing physicians, but also current and future students, ought to be trained to understand and apply genomic information: "without the inclusion of genomic medicine in medical education, physicians will soon not be able to answer their patients' demands" [28].

\section{Speed to market: regulation,} reimbursement \& clinical utility

The speed at which diagnostic innovations are implemented into clinical routine does not exclusively depend on the rate at which they are adopted by practitioner groups, but is also subject to regulatory and reimbursement issues, which can significantly delay a novel technology's speed to market. This can be seen as a major difference between Moore's law in information technology and in diagnostic testing in oncology.

In Europe, for instance, reimbursement, health technology assessment and pricing decisions are subject to national authorities, while regulatory decisions are generally made at the European level [113]. This creates a lack of overall consistency in the fields of technology evaluation, evidence requirements and market access. Furthermore, the regulatory approval of a novel technology in most cases requires data suggesting strong clinical utility. The generation of such data, however, can often only take place after the implementation of a technology. In addition to this, the generation of data on clinical utility is costly and time consuming [103].

The reimbursement of diagnostic technologies thus becomes a lengthy process in which pharmaceutical and diagnostic firms have to determine whether a structure for reimbursement exists - either via a code-based system or coverage through hospitals and laboratories [114]. However, as hospitals and laboratories may not be equipped with sufficient financial resources, they may have to cut back on the number of patients that can be diagnosed appropriately [114].

Against this background, novel approaches towards the assessment of diagnostic technologies are currently being discussed. An example of such an approach is given by risk-sharing schemes, which are increasingly applied [30]. A diagnostic test would initially only be partly reimbursed and would receive full coverage once the clinical value has been demonstrated [31].

The benefits of increasing the speed to market by providing adequate reimbursement structures are not only limited to diagnostic and pharmaceutical firms, but apply to multiple stakeholder groups: physicians are provided with better metrics to identify the best clinical option, patients receive improved diagnosis and treatment, and funds are spent on treatments with the highest response rate [114]. 
As $10-30 \%$ of future drugs are expected to require a companion diagnostic test [106], the absence of appropriate reimbursement schemes is likely to substantially limit the access patients have to innovative therapies [32]. In fact, the lack of appropriate regulatory and reimbursement systems is seen as a major reason why molecular diagnostics have not yet had the uptake and utilization they were expected to have [32].

The increasing implementation of parallel testing technologies, such as multiplex testing, further increases the complexity of the reimbursement situation, as a single test is likely to use probes owned by different diagnostic companies [114]. Efficient and increasingly standardized regulatory and reimbursement structures including improvements in the areas of risk assessment and review criteria are, therefore, becoming more and more critical [113]. They play an essential role in accelerating the speed to market of novel technologies and initiating their diffusion as early as possible.

\section{Conclusion}

Diagnostic testing is a rapidly evolving field with powerful technologies poised to be implemented into clinical routine. Multiplex testing and whole-genome sequencing are associated with major benefits compared with single testing and help overcome the physical constraint posed by increasing biomarker availability. A broad implementation of current technologies, however, enhances the general creation of an overall diagnostics infrastructure. It is, therefore, vital to not only wait for future innovations to arrive, but to widely adopt existing approaches today. To increase the speed to market of innovative technologies and initiate their diffusion at an early stage, it will be necessary to reassess current regulatory and reimbursement schemes, and provide more standardized procedures.

\section{Future perspective}

As the field of diagnostic testing will significantly evolve during the next few years, pathologists

\section{Executive summary}

\section{Background}

- Diagnostic tests represent a crucial technology in personalized cancer care and follow the basic rules of innovation lifecycles.

\section{Technology S-curves}

- Technology S-curves help us to understand how different diagnostic tests evolve and how their performance develops over time.

\section{S-curve 1: single testing}

- Single testing involves the sequential testing of different biomarkers.

\section{Growing biomarker availability}

- The growing availability of biomarkers limits the feasibility of single testing and leads to the aging of this technology.

\section{S-curve 2: parallel testing}

- Parallel testing is less affected by the increasing availability of biomarkers and leads to a more efficient allocation of resources.

- Multiplex assays are one example of parallel testing, analyzing up to 384 biomarkers simultaneously.

"Multiplex assays are increasingly implemented into clinical practice as their feasibility and benefits have been proven in several studies.

\section{S-curve 3: whole-genome sequencing}

- Whole-genome sequencing is a universal diagnostic approach delivering a complete picture of health and disease at an individual level As the 'US $\$ 1000$ genome' has become reality, whole-genome sequencing and genomic medicine are poised to be implemented into clinical routine.

\section{Diffusion of testing technologies}

- Different practitioner groups adopt innovative technologies at a different speed.

- A significant proportion of the market does not succeed in adopting an innovation before it is replaced by a novel technology.

\section{Speed to market: regulation, reimbursement \& clinical utility}

- To increase the speed to market of innovative technologies and initiate their diffusion as early as possible, a reassessment of regulatory and reimbursement schemes is required.

\section{Conclusion}

- Diagnostic testing is a rapidly evolving field with powerful technologies poised to be implemented into clinical routine.

- An early and extensive adoption of single-testing diagnostics sets up the infrastructure to fully benefit from future technologies.

\section{Future perspective}

- Pathologists will play a key role in the process of translating sequencing technologies into mainstream clinical practice.

- It will be inevitable that all stakeholders, including pathologists, clinicians, payers, industry representative, patients and others, make a concerted effort to maximize the value of current and future technologies. 
will play a key role in the process of translating sequencing technologies into mainstream clinical practice [20], which is why their role will have to be redefined. While the question of when the correlation between a specific genetic abnormity and a certain disease justifies a diagnosis is strongly influenced by regulatory agencies and guideline authors, pathologists will increasingly have to deal with the same question by assessing how far the available data suffices to make a clear diagnosis [33]. Although this era has not yet been reached, the prospect of new technologies is already having implications on the obligations and responsibilities of pathologists today [34]. Through an early adoption of innovative technologies, pathologists are capable of facilitating the transition from current to future testing approaches.

It is important to notice that diagnostic testing is embedded into the overall framework of personalized medicine, which is a broad field involving experts from different disciplines. For that reason it will be inevitable that all stakeholders, including pathologists, clinicians, payers, industry representative, patients and others, make a concerted effort to maximize the value of current and future technologies.

As part of this, a trend towards private and public partnerships with academia will increasingly be observed in various countries. Partnerships are frequently an essential part of delivering the promise of personalized medicine and diagnostic testing because no single stakeholder will be able to set all the necessary requirements by himself [35].

Accepting the importance of diagnostic testing today and supporting its evolution in the future will revolutionize the field of oncology and finally lead to a truly personalized approach to medicine.

\section{Disclaimer}

The opinions expressed in this manuscript do not necessarily represent those of Pfizer.

\section{Financial \& competing interests disclosure}

$C$ Lenz is a current employee of Pfizer Deutschland GmbH. JA Steffen was employed at Pfizer until September 2012. The authors have no other relevant affiliations or financial involvement with any organization or entity with a financial interest in or financial conflict with the subject matter or materials discussed in the manuscript apart from those disclosed.

No writing assistance was utilized in the production of this manuscript.

\section{References}

Papers of special note have been highlighted as:

= of interest

"II of considerable interest

1 Wadelius M, Chen LY, Lindh JD et al. The largest prospective warfarin-treated cohort supports genetic forecasting. Blood 113(4), 784-792 (2008).

2 Hung SI, Chung WH, Jee SH et al. Genetic susceptibility to carbamazepine-induced cutaneous adverse drug reactions. Pharmacogenet. Genomics 16(4), 297-306 (2006).

3 Jain KK. Textbook of Personalized Medicine. Springer, NY, USA, 383-393 (2009).

4 Moore G. Cramming more components onto integrated circuits. Electronics 38(8), 114-117 (1965).

5 Williams C, Champion T, Hall I. MGMT. Cengage Learning, Toronto, Canada, 126-127 (2011).

6 Rogers EM. Diffusion of innovations. Free Press, NY, USA (1962).

7 Simmons LA, Dinan MA, Robinson TJ et al. Personalized medicine is more than genomic medicine: confusion over terminology impedes progress towards personalized healthcare. Per. Med. 9(1), 85-91 (2012).
8 Tavares P, Dias L, Palmeiro A et al. Singletest parallel assessment of multiple genetic disorders. Per. Med. 8(3), 375-379 (2011).

- Analyzes the therapeutic and economic benefits of multiplex assays as a diagnostic testing technology.

9 Horn L, Pao W. EML4-ALK: honing in on a new target in non-small-cell lung cancer. J. Clin. Oncol. 27(26), 4232-4235 (2009).

10 Metzler I. Biomarkers and their consequences for the biomedical profession: a social science perspective. Per. Med. 7(4), 407-420 (2010).

- Describes the growing biomarker availability as a trend challenging the approach of companion diagnostics.

11 Hirsch FR, Wynes MW, Gandara DR et al. The tissue is the issue: personalized medicine for non-small cell lung cancer. Clin. Cancer Res. 16(20), 4909-4911 (2010).

12 Ilie M, Hofman P. Pitfalls in lung cancer molecular pathology: how to limit them in routine practice? Curr. Med. Chem. 19(16), 2638-2651 (2012).

13 Thunnissen E, Kerr KM, Herth FJ et al. The challenge of NSCLC diagnosis and predictive analysis on small samples. Practical approach of a working group. Lung Cancer 76(1), 1-18 (2012).
14 Su Z, Ning B, Fang $\mathrm{H}$ et al. Next-generation sequencing and its applications in molecular diagnostics. Expert Rev. Mol. Diagn. 11(3), 333-334 (2011).

15 Tran B, Dancey JE, Kamel-Reid S et al. Cancer genomics: technology, discovery, and translation. J. Clin. Oncol. 30(10), 647-660 (2012).

16 Sequist LV, Heist RS, Shaw AT et al. Implementing multiplexed genotyping of non-small-cell lung cancers into routine clinical practice. Ann Oncol. 22(12), 2616-2624 (2011).

" Underlines the feasibility of multiplex testing in clinical practice.

17 Zander T, Heukamp LC, Bos MC et al. Regional screening network for characterization of the molecular epidemiology of non-small cell lung cancer (NSCLC) and implementation of personalized treatment. J. Clin. Oncol. 30 (Suppl.), Abstract CRA10529 (2012).

18 Saffitz JE. Genomic pathology: a disruptive innovation. Per. Med. 9(3), 237-239 (2012).

19 McDermott U, Downing JR, Stratton MR. Genomics and the continuum of cancer care. N. Engl. J. Med. 364(4), 340-350 (2011).

20 Hall N. Advanced sequencing technologies and their wider impact in microbiology. J. Exp. Biol. 210(Pt 9), 1518-1525 (2007). 
21 Walter MJ, Graubert TA, Dipersio JF et al. Next-generation sequencing of cancer genomes: back to the future. Per. Med. 6(6), 653-662 (2009).

22 Iyer G, Hanrahan AJ, Milowski MI et al. Genome sequencing identifies a basis for everolimus sensitivity. Science 338(6104), 221 (2012).

- Analyzes the potential and benefits of genome sequencing as a diagnostic testing technology.

23 Crawford JM, Aspinall MG. The business value and cost effectiveness of genomic medicine. Per. Med. 9(3), 265-286 (2012).

24 Kahvejian A, Quackenbush J, Thompson JF. What would you do if you could sequence everything? Nat. Biotechnol. 26(10), 1125-1133 (2008).

25 Lindeman N. Molecular diagnostics of lung cancers at the Brigham and Women's Hospital and Dana-Farber Cancer Institute: technology in rapid evolution. Arch. Pathol. Lab. Med. 136(10), 1198-1200 (2012).

26 Tanner NT, Pastis NJ, Sherman C et al. The role of molecular analyses in the era of personalized therapy for advanced NSCLC. Lung Cancer 76(2), 131-137 (2012).

27 McInerney JD, Edelman E, Nissen T et al. Preparing health professionals for individualized medicine. Per. Med. 9(5), 529-537 (2012)

28 Carlberg C. The need for education in personalized medicine. Per. Med. 9(2), 147-150 (2012)

" Points out that the education of health professionals on genomics is a critical driving force behind the successful implementation of personalized medicine.

29 Haspel RL, Atkinson JB, Barr FG et al. TRIG on TRACK: educating pathology residents in genomic medicine. Per. Med. 9(3), 287-293 (2012).

30 Miller I, Ashton-Chess J, Solders $\mathrm{H}$ et al. Market access challenges in the EU for high emdical value diagnostic tests. Per. Med. 8(2), 137-148 (2011)

31 Davis JC, Furstenthal L, Seai AA et al. The microeconomics of personalized medicine: today's challenge and tomorrow's promise. Nat. Rev. Drug Discov. 8(4), 279-286 (2009).
32 Gibbs J. Regulating molecular diagnostic assays: developing a new regulatory structure for a new technology. Expert Rev. Mol. Diagn. 11(4), 367-381 (2011).

33 Korn D. Interview: a perspective on personalized medicine: Dr. David Korn. Per. Med. 9(3), 259-263 (2012).

34 Moch H, Blank PR, Dietel M et al. Personalized cancer medicine and the future of pathology. Virchows Arch. 460 (1), 3-8 (2012).

- Identifies practically relevant implications of new diagnostic testing technologies, including a redefinition of the pathologist's role.

35 Lenz C, Fensch M, Sollano JA. Implementing personalized medicine in clinical practice: country investments and public-private partnerships in Europe. J. Pharmakologie Therapie 1, 3-7 (2012).

\section{Websites}

101 Personalized Medicine Coalition. The case for personalized medicine (2011). www.personalizedmedicinecoalition.org/ about/about-personalized-medicine/the-casefor-personalized-medicine (Accessed 20 August 2012)

102 Business Dictionary. Performance (2012). www.businessdictionary.com/definition/ performance.html (Accessed 21 August 2012)

103 Pletcher MJ, Pignone M. Evaluating the clinical utility of a biomarker: a review for methods estimating health impact. American Heart Association (2011).

http://circ.ahajournals.org/ content/123/10/1116

(Accessed 4 May 2011)

104 von Holleben M, Pani M, Heinemann A. Medizinische Biotechnologie in Deutschland 2011. Boston Consulting Group (2011). www.vfa-bio.de/download/bcg-report-2011broschuere.pdf

(Accessed 30 May 2012)

105 US FDA. BLA 98-0369 Herceptin trastuzumab (1998). www.fda.gov/downloads/Drugs/ DevelopmentApprovalProcess/ HowDrugsareDevelopedandApproved/
ApprovalApplications/

TherapeuticBiologicApplications/

UCM307496.pdf

(Accessed 8 February 2013)

106 Varmus H. Biomarker diagnostics drive personalized medicine \#1 update (2010). http://raygent.com/biograph/biomarkerdiagnostics-drive-personalized-medicine (Accessed 23 May 2012)

107 Ecancer News: the latest oncology headlines. ecancer news (2012). http://ecancer.org/news/2899 (Accessed 8 August 2012)

108 NIH. All about the Human Genome Project (HGP) (2011).

www.genome.gov/10001772/

(Accessed 29 May 2012)

109 National Human Genome Research Institute. DNA sequencing cost (2012). www.genome.gov/SequencingCosts/ (Accessed 22 May 2012)

110 Illumina, MiSeq ${ }^{\circledast}$ system (2013). www.illumina.com/documents/products/ datasheets/datasheet_miseq.pdf (Accessed 3 February 2013)

111 Ion Torrent. Life Technologies: Ion Torrent ${ }^{\mathrm{TM}}$ (2013).

http://blueseq.com/knowledgebank/ sequencing-platforms/life-technologies-iontorrent/

(Accessed 3 February 2013)

112 Arbanas C. Washington University. Decoding cancer patients' genome is powerful diagnostic tool (2011). http://news.wustl.edu/news/Pages/22186. aspx

(Accessed 2 June 2012)

113 Garfield S; Personalized Medicine Coalition. Advancing access to personalized medicine: a comparative assessment of European reimbursement systems (2011). www.personalizedmedicinecoalition.org/sites/ default/files/files/PMC_Europe_ Reimbursement_Paper_Final.pdf (Accessed 2 February 2013)

114 Creighton D. Bridging the hidden hurdle in cancer cures (2011). www.pharmexec.com/pharmexec/article/ articleDetail.jsp?id $=739273$

(Accessed 4 February 2013) 